\title{
The benefit of prothrombin complex concentrate in decreasing neurological deterioration in patients with warfarin-associated intracerebral haemorrhage
}

\author{
WC Fong *, WT Lo, YW Ng, YF Cheung, Gordon CK Wong, HF Ho, John HM Chan, Patrick CK Li
}

\section{A B S T R A C T}

Objective: To compare the outcomes of patients with warfarin-associated intracerebral haemorrhage given different treatments to reverse the effect of anticoagulation.

Design: Historical cohort study.

Setting: A regional hospital in Hong Kong.

Patients: Patients on warfarin who developed intracerebral haemorrhage.

Interventions: Prothrombin complex concentrate versus fresh frozen plasma treatment.

Main outcome measures: The primary outcome measures included the international normalised ratio before and after prothrombin complex concentrate treatment and the neurological deterioration in patients with Glasgow Coma Scale score of more than $8 /$ not intubated/not planned for immediate surgery (target group). Secondary outcome measures were haematoma expansion, 7-day and 30-day mortality rates, and 3-month functional outcome. Safety outcome was the occurrence of a thrombotic event after prothrombin complex concentrate treatment within the index admission.

Results: Among 33 patients with clearly documented time of infusion of prothrombin complex concentrate, and whose international normalised ratio was checked before and after prothrombin complex concentrate treatment, the mean international normalised ratio was reduced from 2.81 to 1.21 within 24 hours. Within the target

group of patients, there was a significantly lower rate of neurological deterioration in the prothrombin complex concentrate group (17.4\% of 23 patients) versus fresh frozen plasma group $(45.5 \%$ of 33 patients) $[\mathrm{P}=0.027]$. In terms of the 7 -day mortality, 30-day mortality, and 3-month functional outcome, prothrombin complex concentrate-treated group showed a favourable trend although the difference did not reach a statistical significance. No patient developed thrombotic complications after prothrombin complex concentrate treatment.

Conclusions: Prothrombin complex concentrates can reverse the warfarin effect of prolonged international normalised ratio in a timely manner. It might better improve the outcome of warfarinassociated intracerebral haemorrhage compared with fresh frozen plasma treatment by reduction in neurological deterioration.
Hong Kong Med J 2014;20:486-94
DOI: $10.12809 / \mathrm{hkmj} 144246$

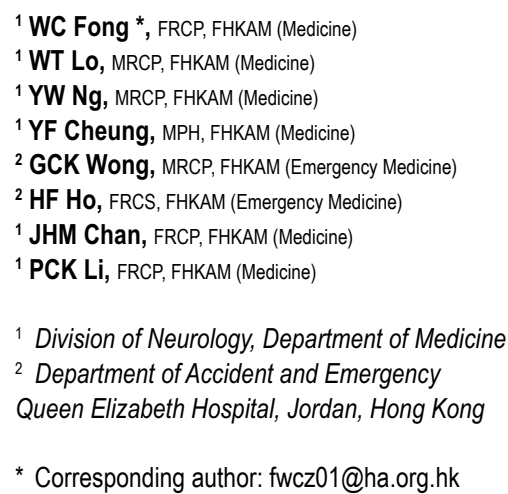

New knowledge added by this study

- Outcome of warfarin-associated intracerebral haemorrhage might be improved by prothrombin complex concentrate treatment.

Implications for clinical practice or policy

- Prothrombin complex concentrate should be considered the first-line reversal agent for patients with warfarinassociated intracerebral haemorrhage unless contra-indicated.

\section{Introduction}

Warfarin-associated intracerebral haemorrhage (ICH) is associated with high mortality of $40 \%$ to $60 \%$. Compared with spontaneous $\mathrm{ICH}$, it has a higher mortality rate and poorer functional outcome. ${ }^{1,2} \mathrm{Up}$ to $50 \%$ of patients will have haematoma expansion within 24 hours, and the haematoma expansion time interval is more prolonged versus those with 
spontaneous $\mathrm{ICH} .{ }^{3}$ Predictors of poor outcome include age, Glasgow Coma Scale score (GCS) on admission, baseline haematoma volume, and haematoma expansion ${ }^{2,4}$; of these, haematoma expansion is the only predictor that can be modified upon admission. Controlling/limiting haematoma expansion, thus, serves as the target for improving the outcome.

Prompt reversal of anticoagulation has been found to decrease haematoma expansion, especially if it can be reversed within 2 hours of admission. ${ }^{5,6}$ The available agents for this include vitamin $\mathrm{K}$, fresh frozen plasma (FFP), prothrombin complex concentrates (PCC), and recombinant factor VII (rFVIIa). Vitamin K should be given to all patients for sustained international normalised ratio (INR) reversal; however, given by itself, it is associated with a slow onset of action. Prothrombin complex concentrate contains factor II, IX, X (3-factor), and some preparations also have factor VII (4-factor). If only 3-factor PCC are available, as in the case of hospitals in Hong Kong, it has been recommended to give additional FFP to replenish factor VII levels. ${ }^{7,8}$ Prothrombin complex concentrates are preferred over FFP alone for warfarin reversal as these can be given quickly without any need for cross-matching of blood or thawing, and have a much smaller volume of infusion, decreasing the risk of volume overload. The onset of action is much faster than FFP and the INR can be reversed in as early as 15 minutes after infusion; in contrast, FFP may take hours for INR reversal. Prothrombin complex concentrates are readily available, are less expensive than rFVIIa, and have a longer half-life than rFVIIa. ${ }^{1}$ Many international societies recommend PCC as the first-line agents for warfarin reversal in emergency situations. ${ }^{9-12}$ Those recommendations are, however, mainly based on rapid reversal of INR by PCC. Currently, there is no prospective, randomised study comparing PCC versus FFP in terms of the longterm functional outcomes. In Hong Kong, PCC are not commonly used for warfarin reversal, probably because these have not been proven to be superior to FFP in large-scale studies, and are associated with a low but definite risk of thrombosis.

Our hospital started formal implementation of PCC protocol for warfarin-associated $\mathrm{ICH}$ in 2011, initially in the Department of Medicine, and subsequently in the Accident and Emergency Department and Department of Neurosurgery (Fig 1) [while PCC were used in 2010, a formal protocol was not implemented until 2011]. The PCC preparation in our hospital was Prothrombinex$\mathrm{HT}$, and was later supplied as Prothrombinex-VF (3-factor; CSL Behring, Broadmeadows, Australia). Our protocol recommends the prompt use of the 3factor PCC with dosage based on INR level, together with intravenous injection of $10 \mathrm{mg}$ vitamin $\mathrm{K}$ and

\section{華法林相關性腦出血患者接受凝血酶原複合物 致減少神經功能惡化的好處 \\ 方榮志、勞慧婷、伍綺華、張暗暉、黃志強、何曉輝、 陳曉明、李頌基}

目的：比較華法林相關性腦出血患者接受各種逆轉抗凝治療法的結 果。

設計：歷史隊列研究。

安排：香港一所分區醫院。

患者：正服用華法林而有腦出血的患者。

干預：以凝血酶原複合物（PCC）或新鮮冷凍血漿（FFP）為基礎的 治療。

主要結果測量：主要療效指標包括接受PCC前後的國際標準化比值 （INR），以及在格拉斯哥昏迷量表評分超過 8 、不插管和沒有計劃立 即進行手術治療的患者 (目標組) 的神經功能惡化情況。次要療效指 標為血腫擴大、七天和三十天死亡率, 以及三個月功能性預後。安全 結果則定義為在該次住院期內接受PCC治療後有血栓形成。

結果 : 在有明確記載接受輸注PCC時間的33名患者中, 他們的INR由 接受治療前的 2.81 降至治療後 24 小時內的 1.21 。目標組內接受PCC 的23名患者出現神經功能惡化的比率明顯較接受FFP的33名患者為低 （17.4\%比 $45.5 \% ； \mathrm{P}=0.027 ）$ 。至於七天和三十天死亡率, 以及三個 月功能性預後方面, 接受PCC的患者有較良好的發展趨勢, 但與接受 FFP患者的差異未達統計顯著性。沒有患者在接受PCC後出現血栓併 發症。

結論：PCC可及時逆轉華法林延長INR的作用。與FFP比較, PCC能 透過減少神經功能惡化的發生, 從而改善華法林相關性腦出血的情 況。

2 units of FFP upon diagnosis, and rechecking INR 30 minutes after administration of PCC; additional warfarin reversal agents are given provided the INR remains high.

The objectives of this study were to review the use of PCC in our hospital (including the use, dosage, complications) and to compare the clinical outcomes with patients given FFP treatment, with the ultimate aim of looking for indirect evidence of benefit of PCC over FFP treatment.

\section{Methods}

This was a retrospective review of patients admitted to Queen Elizabeth Hospital, a tertiary hospital in Hong Kong. Data on patients with ICH and use of warfarin prior to admission were retrieved from the Clinical Data Analysis and Reporting System. Data of patients who were treated with PCC from February 2011 (start of implementation of PCC protocol) to September 2013 were analysed. This was the PCC group. Similarly, data from January 2007 to September 2013 were reviewed and data of patients given treatment other than PCC were analysed. Only patients given FFP were included in the comparison 


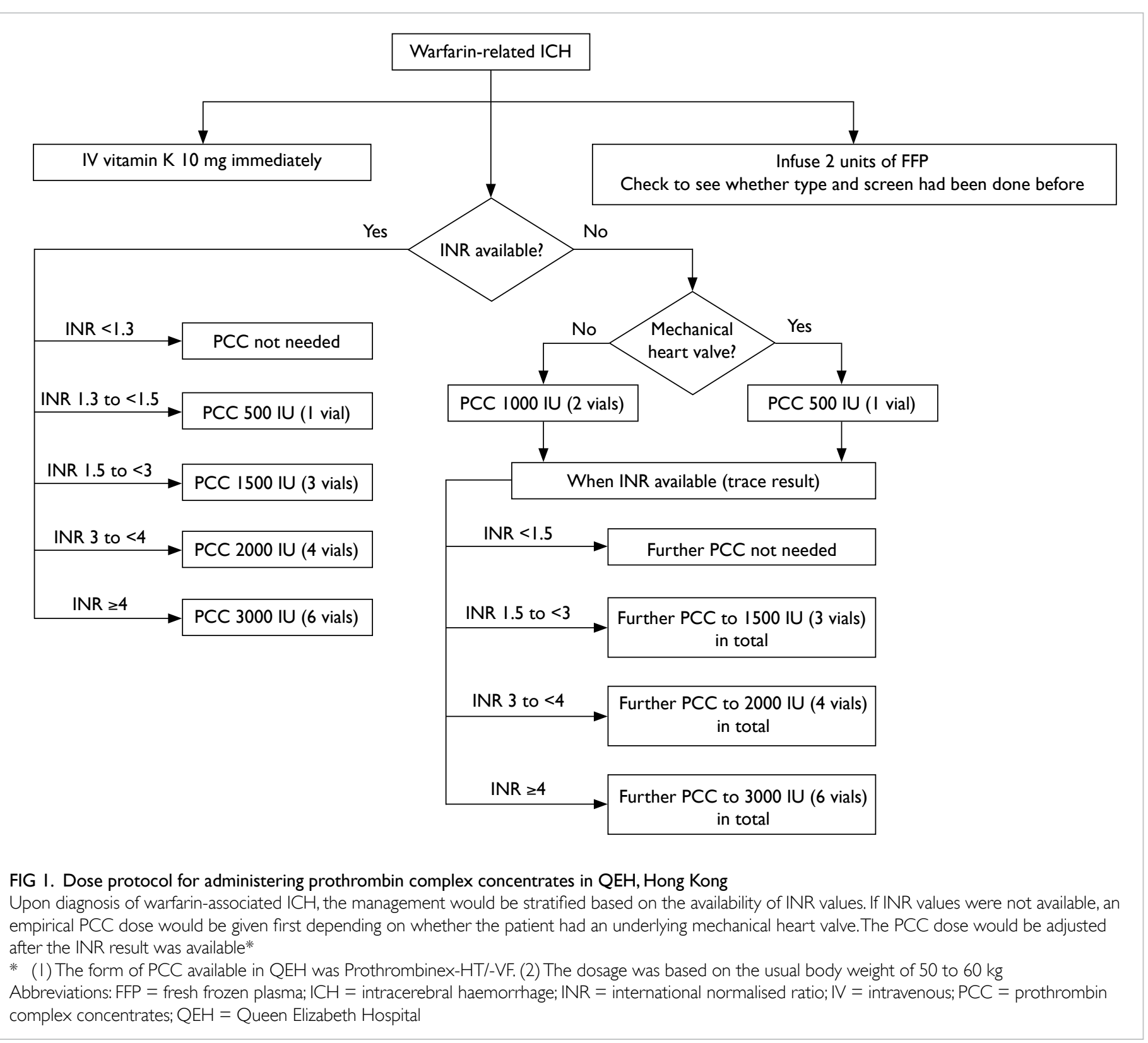

arm. This was the FFP group.

Patients given no reversal agents or solely given vitamin $\mathrm{K}$ were not included in this study. One patient given rFVIIa was excluded. Patients with known or suspected secondary causes of bleeding such as tumour bleeding, underlying subacute bacterial endocarditis, and vasculitis were excluded. Patients with haemorrhagic transformation of infarct, subdural haemorrhage, and subarachnoid haemorrhage were excluded. One patient who was started on warfarin reversal agent in another hospital and later transferred to our hospital after a few days was also excluded.

From the case records, all data including demographics (age, sex, indications for anticoagulation, concomitant use of antiplatelet agent, co-morbidities such as diabetes, hypertension, ischaemic heart disease, prior stroke), clinical state at the time of admission (GCS, limb power), management including reversal agents used (vitamin $\mathrm{K}$, FFP, PCC), intubation, and whether surgery had been performed were reviewed. Laboratory data (INR on admission and after PCC use) and radiological data (baseline haematoma volume and follow-up computed tomography [CT] brain scans) were reviewed from the Electronic Patient Record system. The CT scans were reviewed by two authors who were not blinded to the treatment information, while the intracerebral haematoma volume was calculated based on the $\mathrm{ABC} / 2$ score. ${ }^{13}$ Haematoma volume within the ventricular extension was estimated by using the intraventricular haemorrhage 
score. ${ }^{14}$

The primary outcome measure was neurological deterioration, defined as $\geq 2$-point decrease in GCS or limb power. This was selectively studied in patients with an initial GCS of more than 8 , who were not intubated and who were not initially considered for surgery upon diagnosis of $\mathrm{ICH}$, ie those who were initially selected for medical treatment (target group). The rationale for this was that the patients who have the maximum benefit from rapid reversal agents are those with initial good GCS, for whom the agents can prevent further deterioration. Patients with poor GCS due to large ICH upon presentation probably do not benefit much from rapid reversal. The secondary clinical outcome measures were haematoma expansion (defined as $>33 \%$ increase in haematoma volume), ${ }^{15} 7$-day and 30-day mortality rates, and modified Rankin scale (mRS) on 3 months of follow-up.

Statistical analysis was performed using the Statistical Package for the Social Sciences (Windows version 20; SPSS Inc, Chicago [IL], US). Proportion between groups was compared using the Fisher's exact test, and continuous variables were compared using the Wilcoxon rank sum test. P value was considered significant if it was less than 0.05 .

\section{Results}

Figure 2 shows the recruitment of patients with warfarin-associated $\mathrm{ICH}$ identified before and after protocol implementation and the subsequent formation of cohorts for analysis.

\section{Analysis of use of prothrombin complex concentrates (from February 2011 to September 2013 after implementation of protocol)}

Of 70 patients who had warfarin-associated $\mathrm{ICH}$, 61 were given $\mathrm{PCC}$, and nine patients were not given PCC for the following reasons: too ill for undergoing $\mathrm{CT}$ brain when stroke was suspected, and stroke confirmed only afterwards $(n=1)$; nearly moribund state on admission $(n=6$; one of these had concomitant allergy to FFP and two had recent pulmonary embolism/deep vein thrombosis); and small ICH size $\left(0.07 \mathrm{~cm}^{3}\right)$ and symptom onset more than 1 week ago with a mechanical heart valve and carotid stent $(n=1)$. No reason was documented for one patient.

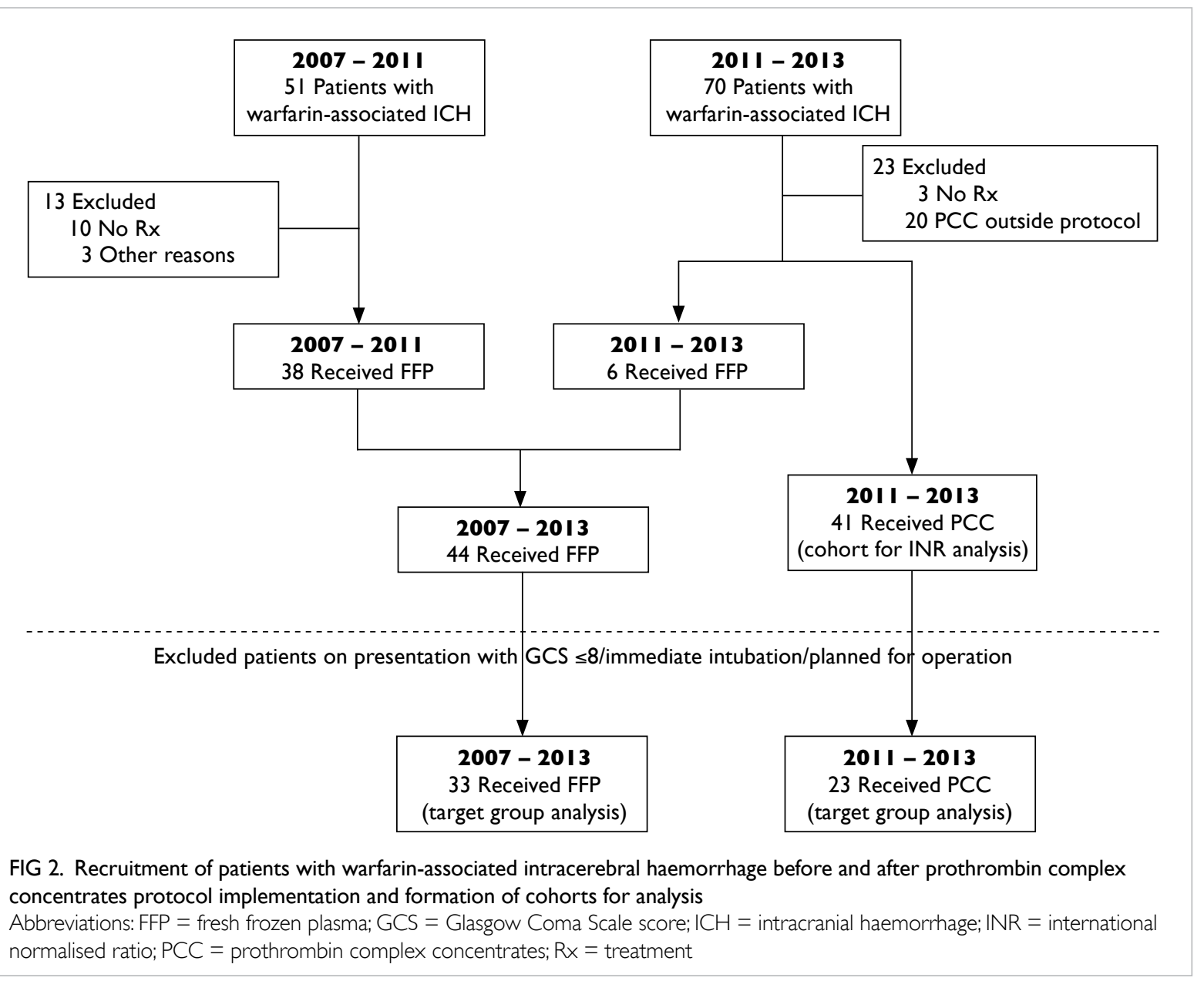


Only 41 out of the 61 patients given PCC from February 2011 to September 2013 were included in the PCC group for INR analysis and for comparison with the FFP group. Twenty patients were excluded because the reversal treatment regimen had been modified by the treating clinicians and was different from our protocol $(n=17)$, and patient records were kept by other parties and we failed to confirm the dose given $(n=3)$.

Their mean age was 74 years and their mean GCS on presentation was 11 . A total of 18 patients had GCS of $\leq 8$ /were immediately intubated/planned for operation; 16 patients had GCS of $>13$ on presentation.

\section{Dosage of prothrombin complex concentrates and reversal of warfarin effect}

Among the 41 patients, time of infusion of PCC was clearly documented and INR was checked before and within 24 hours after PCC infusion in 33 patients. The mean pre-PCC INR was 2.81 (interquartile range [IQR], 1.95-2.92) and the mean post-PCC INR was 1.21 (IQR, 1.09-1.39). In 29/33 (87.9\%) patients, INR was reversed to $\leq 1.4$ in the post-PCC INR test. The mean time of checking the INR post-PCC was 4 hours and 15 minutes. In 11 patients, INR was checked within 2 hours after PCC had been given. Nine $(81.8 \%)$ out of these 11 patients achieved INR of $\leq 1.4$ within 2 hours, with mean pre-PCC INR being 2.43 (IQR, 2.05-2.73) and mean post-PCC INR being 1.27 (IQR, 1.17-1.40).

\section{Haematoma expansion}

One patient underwent brain imaging in a private hospital and, thus, the baseline haematoma volume could not be traced. Of the 41 patients, 32 underwent follow-up CT brain within 7 days (within 48 hours in 27 patients, within 2 to 3 days in two patients, and between 3 and 7 days in three patients). Of these 32 patients, seven $(21.9 \%)$ had haematoma expansion. In three out of these seven patients, INR was checked within 2 hours and corrected to $\leq 1.4$. The INR was not rechecked promptly in the remaining four patients.

\section{Thromboembolic complications}

One patient had an ischaemic infarct 19 days after $\mathrm{ICH}$. This patient had underlying atrial fibrillation and warfarin was withheld after admission. There were, otherwise, no patients with recorded thromboembolic events within the index admission in the whole group of 61 patients given PCC.

\section{Comparison between two groups receiving either prothrombin complex concentrates or fresh frozen plasma}

Fifty-seven patients identified from January 2007 to
September 2013 had warfarin-associated ICH which was not treated with PCC. Of these, only 44 patients were finally included in our analysis for comparison with the PCC group. Ten patients had not been given any warfarin reversal agents. Three other patients were excluded-one had started warfarin reversal agents in another hospital and was later transferred to our hospital days (for geographical reasons); one was given rFVIIa; and in one patient the diagnosis of $\mathrm{ICH}$ was made retrospectively, after cardiac arrest.

\section{Mortality and functional outcome in the whole group}

The outcomes of both groups were similar in terms of 7-day and 30-day mortality rates and 3-month mRS (Table 1). However, there were differences in terms of proportion of target group patients. In the PCC group, $43.9 \%$ had GCS of $\leq 8 /$ intubated/already planned for operation on admission; in contrast, only $25 \%$ from the FFP group were in this group (Table 2). Since patients with poor GCS were associated with poor outcomes, such baseline differences probably abolished the treatment effect of PCC over FFP.

\section{Outcome analysis in the target group}

As more than $40 \%$ of our patients had a GCS of $\leq 8$ on presentation, and were destined to have a poor functional outcome, we selectively compared the outcome in patients of the target group (GCS $>8$, not intubated, not initially considered for surgery upon diagnosis of $\mathrm{ICH}$ ) as planned. Their baseline characteristics were similar, apart from age which was higher in the PCC group (Table 3). The neurological deterioration rate was significantly lower in those given PCC $(n=4 / 23 ; 17.4 \%)$ versus those given FFP $(n=15 / 33 ; 45.5 \% ; P=0.027)$. After adjustment for age by logistic regression, PCC treatment was independently associated with lower risk of neurological deterioration (odds ratio $=0.256$; 95\% confidence interval, 0.069-0.956; $\mathrm{P}=0.043)$.

Among patients who underwent follow-up CT scan, haematoma expansion was lower in patients

TABLE I. Outcomes of the two groups

\begin{tabular}{lrrr}
\hline & PCC (n=41) & FFP (n=44) & P value \\
\hline 7-Day mortality & $14(34.1 \%)$ & $15(34.1 \%)$ & 0.588 \\
30-Day mortality & $17(41.5 \%)$ & $21(47.7 \%)$ & 0.359 \\
3-Month mRS & & & \\
$0-3$ & $16(39.0 \%)$ & $14(31.8 \%)$ & 0.737 \\
$4-5$ & $6(14.6 \%)$ & $6(13.6 \%)$ & \\
6 & $19(46.3 \%)$ & $24(54.5 \%)$ & \\
\hline
\end{tabular}

Abbreviations: FFP = fresh frozen plasma; $m R S=$ modified Rankin scale; PCC = prothrombin complex concentrates 
TABLE 2. Comparison of baseline data between the two groups

\begin{tabular}{|c|c|c|c|}
\hline & $\operatorname{PCC}(n=41)$ & FFP $(n=44)$ & $P$ value \\
\hline Mean age (years) & 74 & 70 & 0.174 \\
\hline Male gender & $23(56.1 \%)$ & $19(43.2 \%)$ & 0.281 \\
\hline Mean GCS on presentation & 11 & 12 & 0.191 \\
\hline $\begin{array}{l}\text { Patients with GCS } \leq 8 \text {, immediate intubation/planned for } \\
\text { operation on presentation }\end{array}$ & 18 (43.9\%) & $11(25.0 \%)$ & 0.073 \\
\hline Median baseline haematoma volume $\left(\mathrm{cm}^{3}\right)$ & 21.0 & 19.9 & 0.123 \\
\hline Median INR on admission & 2.40 & 2.76 & 0.820 \\
\hline
\end{tabular}

Abbreviations: FFP = fresh frozen plasma; GCS = Glasgow Coma Scale score; INR = international normalised ratio; PCC = prothrombin complex concentrates

TABLE 3. Baseline and outcome comparison between different treatments in the target group

\begin{tabular}{|c|c|c|c|}
\hline & $\operatorname{PCC}(n=23)$ & FFP $(n=33)$ & $P$ value \\
\hline Mean age (years) & 74 & 67 & 0.033 \\
\hline Male gender & $10 / 23(43.5 \%)$ & $15 / 33(45.5 \%)$ & 0.787 \\
\hline Mean GCS on presentation & 14 & 14 & 0.540 \\
\hline Median baseline haematoma volume $\left(\mathrm{cm}^{3}\right)$ & 11.0 & 14.9 & 0.506 \\
\hline Median INR on admission & 2.48 & 2.68 & 0.653 \\
\hline \multicolumn{4}{|l|}{ Co-morbidities } \\
\hline DM & $4(17.4 \%)$ & $7(21.2 \%)$ & 0.746 \\
\hline $\mathrm{HT}$ & $13(56.5 \%)$ & $16(48.5 \%)$ & 0.596 \\
\hline IHD & $6(26.1 \%)$ & $10(30.3 \%)$ & 0.773 \\
\hline Previous CVA & $8(34.8 \%)$ & $13(39.4 \%)$ & 0.785 \\
\hline Antiplatelet use & $3(13 \%)$ & $8(24.2 \%)$ & 0.496 \\
\hline \multicolumn{4}{|l|}{ Outcome } \\
\hline Neurological deterioration & $4(17.4 \%)$ & $15(45.5 \%)$ & 0.027 \\
\hline Haematoma expansion & $3 / 19(15.8 \%)$ & $10 / 25(40.0 \%)$ & 0.078 \\
\hline 7-Day mortality & $2(8.7 \%)$ & $4(12.1 \%)$ & 0.798 \\
\hline 30-Day mortality & $4(17.4 \%)$ & $10(30.3 \%)$ & 0.218 \\
\hline \multicolumn{4}{|l|}{ 3-Month mRS } \\
\hline $0-3$ & $14(60.9 \%)$ & $14(44.4 \%)$ & 0.368 \\
\hline $4-5$ & $3(13.0 \%)$ & $6(18.2 \%)$ & \\
\hline 6 & $6(26.1 \%)$ & $13(39.4 \%)$ & \\
\hline
\end{tabular}

Abbreviations: CVA = cerebrovascular accident; DM = diabetes mellitus; FFP = fresh frozen plasma; GCS = Glasgow Coma Scale score; $\mathrm{HT}$ = hypertension; IHD = ischaemic heart disease; INR = international normalised ratio; $\mathrm{mRS}=$ modified Rankin scale; $\mathrm{PCC}=$ prothrombin complex concentrates

given PCC for warfarin reversal $(\mathrm{n}=3 / 19 ; 15.8 \%)$ versus those given FFP $(\mathrm{n}=10 / 25 ; 40.0 \% ; \mathrm{P}=0.078)$. Within the target group, the 7-day and 30-day mortality rates were lower in PCC-treated patients (with PCC: $8.7 \%$ and 17.4\%; with FFP: $12.1 \%$ and $30.3 \%$, respectively) but the differences were not significant (Table 3). More patients were able to walk without assistance on follow-up at 3 months in the PCC group versus the FFP group, without any significant increase in dependency.

\section{Additional analysis of all patients treated with prothrombin complex concentrates}

Of 61 patients given PCC, 20 were excluded from target group analysis because they had not been given PCC according to the protocol (in the form of PCC dose variation or without concomitant FFP). Since there might be a potential possibility of selection bias with one third of the patients excluded from analysis, we repeated the analysis by including all the 61 patients. Among the 20 additional patients, 
12 patients fulfilled the criteria of target group (GCS $>8$, not intubated, not initially considered for surgery upon diagnosis of $\mathrm{ICH})$. Hence, the number of PCC patients in the target group was increased from 23 to 35 . On comparison between all patients given PCC, irrespective of whether it was given according to the protocol $(\mathrm{n}=35)$ and patients given FFP $(\mathrm{n}=33)$, the neurological deterioration rate remained significantly less in the PCC group $(n=7 / 35 ; 20 \%$ vs $\mathrm{n}=15 / 33 ; 45.5 \% ; \mathrm{P}=0.023)$. The haematoma expansion rate was $21.9 \%(\mathrm{n}=7 / 32)$ in the PCC group, and $40 \%$ $(\mathrm{n}=10 / 25)$ in the FFP group $(\mathrm{P}=0.117)$. There were no significant differences in the 7-day, 30-day mortality rates, and 30-day functional outcome.

\section{Discussion}

Warfarin-associated $\mathrm{ICH}$ is associated with a poor outcome and high mortality, and different studies have demonstrated that haematoma expansion is a causative factor. Prompt reversal of anticoagulation has been demonstrated to decrease haematoma expansion, and improve outcome. ${ }^{16}$ In Huttner et al's study, ${ }^{5}$ reversal of INR within 2 hours was associated with less haematoma expansion, and more patients could achieve this with PCC than with FFP. Our study similarly showed that PCC given according to our protocol could adequately reverse INR in a timely manner.

However, a significant improvement in longterm outcomes and decrease in mortality compared to FFP treatment were not well demonstrated in our study; these findings are also not observed in data from published studies. This is partly due to the difficulty in recruiting large number of patients to demonstrate a meaningful difference in outcome. ${ }^{17}$ Furthermore, we postulate that not all patients will benefit from rapid reversal of INR by PCC as compared with FFP treatment. Patients with low level of consciousness are associated with poor outcome, ${ }^{4}$ which might not be improved no matter what kind of reversal treatment is implemented. In order to demonstrate improvement in clinical outcome, studies should stratify patients according to the neurological state on admission or depending on whether surgery has been performed, both of which will affect interpretation of the outcome.

On analysis of the whole PCC group irrespective of GCS, the 30 -day mortality rate was $41.5 \%$ in our study. The 30-day mortality rate among those with GCS of $\leq 8$, intubated or planned for surgery on presentation was $72.2 \%$ ( $\mathrm{n}=13 / 18$ patients), whereas it was $17.4 \%(n=4 / 23$ patients) in those in the target group. As the mortality rate and functional outcome are confounded by the baseline GCS level and also by whether surgery has been done and the outcome of the surgical procedure, we targeted our study on the incidence of neurological deterioration for patients with a fair-to-good admission GCS and who had initially decided to receive conservative medical treatment. This was the target group of patients who was likely to benefit the most from timely treatment in terms of decreased chances of haematoma expansion and neurological deterioration and subsequent poor functional outcome and death. Our study showed that PCC treatment did decrease the risk of deterioration significantly. And to the best of our knowledge, it is the first study to demonstrate that the potential benefit of PCC over FFP is derived from patients with GCS of $>8$ when treatment is started. Such benefit in the reduction of neurological deterioration probably accounted for the trend towards a lower mortality rate and better functional outcome (mRS, 0-3) in the PCC group compared with the FFP group, even though the result was not statistically significant (which might be related to the small sample size). Equally important is the finding that PCC use was not associated with a significant increase in dependency on follow-up at 3 months. This is an important finding as it suggests that decreased mortality with PCC treatment is not associated with an increase in the number of patients in severely dependent state.

It is important to recognise that haematoma expansion and deterioration can occur even in patients with small haematomas, with the chances of neurological deterioration being higher and the duration more prolonged compared with those with spontaneous ICH. ${ }^{3}$ We postulated that patients with small haematomas or stable GCS are the ones who would benefit most from PCC treatment as it can reduce the chances of subsequent deterioration due to haematoma expansion if the bleeding tendency is not rapidly reversed. Treatment should be initiated as soon as possible before any haematoma expansion develops. Initiation of PCC treatment at accident and emergency level, even before INR result is available (as in our protocol), is probably an important factor for improving the outcome of our patients.

The main concern in the use of PCC instead of FFP is the associated risk of thrombosis. In our study, one patient developed an acute ischaemic stroke 19 days after the ICH episode. The elimination halflives of factor II, factor IX, and factor X within the Prothrombinex-VF are 60 hours, 20 hours, and 30 hours, respectively. ${ }^{18}$ Hence, the ischaemic stroke was likely related to the underlying atrial fibrillation rather than to PCC treatment as the time interval between its use and the ischaemic stroke was more than five half-lives of PCC. However, in view of the small sample size of our study, the safety of PCC treatment with our protocol cannot be definitely demonstrated. From other reviews, the incidence of thrombotic events was about $1 \%$, including deep vein thrombosis, pulmonary embolism, myocardial infarction, and ischaemic stroke but this risk of thrombosis was also related to the underlying 
indication of anticoagulation in the first place. ${ }^{11,18,19}$ Also, the risk may be lower with newer preparations of PCC. ${ }^{18}$ In view of this low thrombotic risk and high risk of haematoma expansion, the benefit of PCC for rapid reversal of anticoagulation effect probably overweighs the risk from its thrombogenicity, except in the presence of contra-indications for PCC treatment, namely evidence of active thrombosis or disseminated intravascular coagulation. Patients treated with PCC should be observed closely for symptoms or signs of thrombosis, embolism, myocardial infarction, ischaemic stroke, and disseminated intravascular coagulation.

\section{Limitations}

The most important limitation is that it is a retrospective comparative study of two cohorts, instead of a randomised study. Although FFP was the only treatment available for patients before the introduction of PCC protocol, and PCC was given to most patients after the protocol was implemented; potential bias in the selection of treatment might still occur. Some patients might be excluded from PCC or any treatment because of their moribund status. Also, for this study of two sequential cohorts, there might be potential bias in that the standard of acute care of $\mathrm{ICH}$ might have improved over this 7-year period, leading to better outcomes in the PCC treatment group.

The other limitation is that the data collected were neither complete nor standardised due to the retrospective nature of study design. International normalised ratios were not checked regularly at fixed time intervals post-PCC; similarly, followup CT brain scans were not performed in all patients. Thus, the number of patients available for analysing the adequacy of prompt INR reversal and haematoma expansion was not large. Although the PCC protocol recommended measuring the INR 30 minutes after PCC administration, it was not always performed in a busy clinical setting. Patients who were regarded stable or too unstable might not have undertaken follow-up CT brain to look for haematoma expansion. Bias might also have occurred while measuring haematoma volumes since the raters were not blinded to the treatment given and volumetric analysis of haematoma size was not available. As a result, the validity in assessment of INR reversal and haematoma expansion was diminished. In view of the potential selection and measurement bias inherited from the study design, the favourable trend of the PCC treatment should be interpreted with great caution. Similarly, the retrospective design of the study did not allow pre-specified investigations to reveal the potential thrombotic complications of PCC. It was unreliable to diagnose those complications based on review of case notes alone, especially in such a group of $\mathrm{ICH}$ patients with impaired conscious level. As a result, the safety of PCC treatment remained unconfirmed in this study.

\section{Conclusions}

The current study suggests that the use of PCC in the management of warfarin-associated $\mathrm{ICH}$ can promptly normalise the INR. It showed a trend of outcome improvement in terms of reduction in the risk of subsequent neurological deterioration, without an increase in dependency. Whilst we await results from an ongoing randomised study ${ }^{20}$ to provide us with high-level evidence, PCC should be considered the first-line reversal agent for patients with warfarin-associated $\mathrm{ICH}$ after its potential benefit has been weighed against its small but definite risk of thromboembolism.

\section{References}

1. Aguilar MI, Hart RG, Kase CS, et al. Treatment of warfarinassociated intracerebral hemorrhage: literature review and expert opinion. Mayo Clin Proc 2007;82:82-92.

2. Cucchiara B, Messe S, Sansing L, Kasner S, Lyden P; CHANT Investigators. Hematoma growth in oral anticoagulant related intracerebral hemorrhage. Stroke 2008;39:2993-6.

3. Flibotte JJ, Hagan N, O’Donnell J, Greenberg SM, Rosand J. Warfarin, hematoma expansion, and outcome of intracerebral hemorrhage. Neurology 2004;63:1059-64.

4. Zubkov AY, Mandrekar JN, Claassen DO, Manno EM, Wijdicks EF, Rabinstein AA. Predictors of outcome in warfarin-related intracerebral hemorrhage. Arch Neurol 2008;65:1320-5.

5. Huttner HB, Schellinger PD, Hartmann M, et al. Hematoma growth and outcome in treated neurocritical care patients with intracerebral hemorrhage related to oral anticoagulant therapy: comparison of acute treatment strategies using vitamin $\mathrm{K}$, fresh frozen plasma, and prothrombin complex concentrates. Stroke 2006;37:1465-70.

6. Yasaka M, Minematsu K, Naritomi H, Sakata T, Yamaguchi T. Predisposing factors for enlargement of intracerebral hemorrhage in patients treated with warfarin. Thromb Haemost 2003;89:278-83.

7. Holland L, Warkentin TE, Refaai M, Crowther MA, Johnston MA, Sarode R. Suboptimal effect of a threefactor prothrombin complex concentrate (Profilnine-SD) in correcting supratherapeutic international normalized ratio due to warfarin overdose. Transfusion 2009;49:11717.

8. Franchini M, Lippi G. Prothrombin complex concentrates: an update. Blood Transfus 2010;8:149-54.

9. Baglin TP, Keeling DM, Watson HG; British Committee for Standards in Haematology. Guidelines on oral anticoagulation (warfarin): third edition-2005 update. $\mathrm{Br}$ J Haematol 2006;132:277-85.

10. Baker RI, Coughlin PB, Gallus AS, Harper PL, Salem HH, Wood EM; Warfarin Reversal Consensus Group. Warfarin reversal: consensus guidelines, on behalf of the Australasian Society of Thrombosis and Haemostasis. Med J Aust 2004;181:492-7.

11. Steiner T, Kaste M, Forsting M, et al. Recommendations 
for the management of intracranial haemorrhagepart I: spontaneous intracerebral haemorrhage. The European Stroke Initiative Writing Committee and the Writing Committee for the EUSI Executive Committee. Cerebrovasc Dis 2006;22:294-316.

12. Liumbruno G, Bennardello F, Lattanzio A, Piccoli P, Rossetti G; Italian Society of Transfusion Medicine and Immunohaematology (SIMTI) Working Party. Recommendations for the use of antithrombin concentrates and prothrombin complex concentrates. Blood Transfus 2009;7:325-34.

13. Kothari RU, Brott T, Broderick JP, et al. The ABCs of measuring intracerebral hemorrhage volumes. Stroke 1996;27:1304-5.

14. Hallevi H, Dar NS, Barreto AD, et al. The IVH score: a novel tool for estimating intraventricular hemorrhage volume: clinical and research implications. Crit Care Med 2009;37:969-74, e1.

15. Brott T, Broderick J, Kothari R, et al. Early hemorrhage growth in patients with intracerebral hemorrhage. Stroke 1997;28:1-5.

16. Kuwashiro T, Yasaka M, Itabashi $R$, et al. Effect of prothrombin complex concentrate on hematoma enlargement and clinical outcome in patients with anticoagulant-associated intracerebral hemorrhage. Cerebrovasc Dis 2011;31:170-6.

17. Flaherty ML, Adeoye O, Sekar P, et al. The challenge of designing a treatment trial for warfarin-associated intracerebral hemorrhage. Stroke 2009;40:1738-42.

18. Sørensen B, Spahn DR, Innerhofer P, Spannagl M, Rossaint R. Clinical review: Prothrombin complex concentratesevaluation of safety and thrombogenicity. Crit Care 2011;15:201.

19. Dentali F, Marchesi C, Pierfranceschi MG, et al. Safety of prothrombin complex concentrates for rapid anticoagulation reversal of vitamin $\mathrm{K}$ antagonists. A metaanalysis. Thromb Haemost 2011;106:429-38.

20. Steiner T, Freiberger A, Griebe M, et al. International normalised ratio normalisation in patients with coumarinrelated intracranial haemorrhages-the INCH trial: a randomised controlled multicentre trial to compare safety and preliminary efficacy of fresh frozen plasma and prothrombin complex-study design and protocol. Int J Stroke 2011;6:271-7. 